\section{PWE-092 PSYCHOSOCIAL IMPACT OF FOOD AND NUTRITION IN PEOPLE WITH IBD: A OUALITATIVE STUDY}

doi:10.1136/gutjnl-2013-304907.380

1." L D Hughes, ${ }^{2} \mathrm{~J} O$ Lindsay, ${ }^{3,4} \mathrm{M} \mathrm{C}$ Lomer, ${ }^{5} \mathrm{~S}$ Ayis, ${ }^{4} \mathrm{~L}$ King, ${ }^{5} \mathrm{M}$ Morgan, ${ }^{4} \mathrm{~K}$ Whelan. ${ }^{1} / O P$, King's College London (KCL); ${ }^{2}$ Gastroenterology, Barts Health NHS Trust; ${ }^{3}$ Nutrition \& Dietetics, Guy's \& St Thomas' NHS Foundation Trust; ${ }^{4}$ DNS: ${ }^{5}$ PCPHS, KCL, London, UK

Introduction Food and eating can be a source of pleasure, means of social interaction and peer acceptance. Having Inflammatory Bowel Disease (IBD) may alter these psychosocial factors because of painful or embarrassing symptoms and/or undernutrition resulting in activity limitation. However, little is currently known about the impact of IBD on the psychosocial factors of food and quality of life. This study aimed to determine patients' experiences of the social and psychological impacts of food on people with IBD.

Methods Semi-structured interviews were carried out with 28 patients regarding their experiences of food and eating in relation to their IBD. Interviews were recorded and transcribed verbatim. Concepts were labelled through line by line coding using a constant comparative approach based on grounded theory

Results Mean age and BMI were 36.9 years and 23.1, respectively, $46 \%$ were men, $57 \%$ had Crohn's Disease (CD) and 29\% had had IBD-related surgery. Median (range) disease duration was 6.5 years (1-40). Emerging themes were: (1)Trigger foods "I'm not sure what flares up my IBD"; (2)Family \& friends "I don't have meals with my friends"; (3)Eating in restaurants "Going to restaurants is really difficult because of my IBD"; (4)Toilet issues "I have to go to the toilet whenever I eat" and "I eat less so I have to go to the toilet less"; (5)Practicalities of cooking \& shopping "Cooking different meals for my IBD upsets me" and "It takes longer to shop for food because I read the labels "; and (6) Emotions connected with eating "I'm hard on myself when it comes to coping with eating \& drinking" and "I don't want to be the one that kills the fun for everyone." Feelings of embarrassment, guilt, burden and disappointment around eating were evident for many patients. There were also positive comments around control, adaptive eating and knowledge and support for patients who have developed a successful eating regimen

Conclusion The interviews identified psychosocial issues relating to food and drink for IBD patients whilst their disease was active. However, patients, (especially CD) experienced issues that impacted on their daily eating and drinking as well as social relationships because of self-imposed dietary restraints even during quiescence. The problems and strategies used by patients, particularly during remission, were not known and highlight important areas to be targeted to improve quality of life. Further work is planned to develop a food-related quality of life questionnaire that can be used in the clinical and research setting

Disclosure of Interest None Declared.

\section{PWE-093 SCREENING WITH HOLOTRANSCOBALAMIN IS SUPERIOR TO SERUM B12 IN IDENTIFYING VITAMIN B12 DEFICIENCY IN PATIENTS WITH CROHN'S DISEASE}

doi:10.1136/gutjnl-2013-304907.381

1." M G Ward, 'V C Kariyawasam, 'P A Blaker, ' $\mathrm{K}$ V Patel, 'R M Goel, ${ }^{2} \mathrm{~A}$ SobczynskaMalefora, ${ }^{3} \mathrm{~A}$ Ajaegbu, ${ }^{2} \mathrm{D}$ J Harrington, 'S H Anderson, ' $\mathrm{J}$ D Sanderson, 'P M Irving 'Gastroenterology, Guy's and St Thomas' NHS Foundation Trust; ' ${ }^{T h e}$ Nutristasis Unit; ${ }^{3}$ Diagnostic Haemostasis \& Thombosis, GSTS Pathology, London, UK

Introduction Risk factors for vitamin B12 deficiency in patients with Crohn's disease (CD) include ileal disease and previous ileal resections. Screening for B12 deficiency is traditionally through serum B12 which is relatively insensitive. Holotranscobalamin
(holoTC) is a test that measures the metabolically active fraction of B12 available for cellular uptake and has been shown to perform better than traditional testing in identifying patients with functional B12 deficiency. We hypothesised that holoTC would identify B12 deficiency in patients with CD deemed to be B12 replete on traditional testing and sought to identify prevalence and risk factors within this population.

Methods Prospective study of consecutive patients with CD Patients receiving B12 supplementation were excluded. Patients underwent paired serum B12 and HoloTC testing. Serum B12 $<107 \mathrm{pmol} / \mathrm{L}$ or HoloTC $<25 \mathrm{pmol} / \mathrm{L}$ was defined as B12 deficient. Intermediate HoloTC values between $25 \mathrm{pmol} / \mathrm{L}$ and $50 \mathrm{pmol} / \mathrm{L}$ underwent further assessment with methylmalonic acid (MMA), considered the gold standard in metabolic B12 deficiency. MMA > 280nmol/L in patients < 65 years of age and > $360 \mathrm{nmol} / \mathrm{L}$ in patients $>65$ years of age confirmed B12 deficiency. Risk factors for B12 deficiency were examined including Montreal classification, surgical history and the presence of ileal inflammation or stricture.

Results 70 patients who were not receiving B12 supplementation were included, (37 (53\%) male, median age 37.5 years (IOR 28-47)). Disease location was ileal in 19 (27\%), colonic in 19 (27\%), ileocolonic in 32 (46\%). 27 (39\%) had undergone surgery, 22 (31\%) an ileal resection.

18 (26\%) were B12 deficient using HoloTC; 8 (11.5\%) on HoloTC alone and $10(14.5 \%)$ after MMA analysis on intermediate HoloTC results. Serum B12 testing identified 4 (5.7\%) patients with B12 deficiency; 2 were functionally B12 deficient with HoloTC alone and 2 were replete when assessed by MMA. Ileal resection length $>$ $30 \mathrm{~cm}$ (OR 5.3, 95\% CI 2.6-10.8, p < 0.0001), ileal inflammation (OR 11.3, 95\% CI 3.48-36.9, p < 0.0001), ileal stricture (OR 6.1, 95\% CI 2.8-13.7, $p<0.0001$ ) and ileal resection (OR 5.0, 95\% CI 2.3-10.7, $\mathrm{p}<0.0001)$ were significant predictors of B12 deficiency on univariate analysis.

Conclusion HoloTC identifies vitamin B12 deficiency in a significant percentage of patients with $\mathrm{CD}$ otherwise considered replete on traditional testing. In addition serum B12 testing identifies patients who are not functionally deficient. Active ileal disease, ileal resection and ileal resection $>30 \mathrm{~cm}$ were significant predictors of vitamin B12 deficiency.

Disclosure of Interest None Declared.

\section{PWE-094 THE PREVALENCE OF ANAEMIA IN INFLAMMATORY BOWEL DISEASE IN RELATION TO DISEASE ACTIVITY, AS STRATIFIED BY FAECAL CALPROTECTIN}

doi:10.1136/gutjnl-2013-304907.382

1," M Muscat, 'N A Kennedy, ${ }^{1} \mathrm{~J}$ Chang, ${ }^{1} \mathrm{~J}$ Satsangi, ${ }^{\mathrm{I}} \mathrm{D}$ Arnott, ${ }^{2} \mathrm{~K}$ Kingstone, ${ }^{1} \mathrm{C}$ W Lees 'Gastrointestinal Unit; ${ }^{2}$ Clinical biochemistry, Western General Hospital, Edinburgh, UK

Introduction The prevalence of anaemia in IBD varies significantly between published studies, ranging from 6-74\%. However, underlying disease activity, a potential explanation for this variability, has not been accurately correlated with the frequency of anaemia to date. Faecal calprotectin (FC) is a surrogate marker of underlying mucosal inflammation. The objective of this study was to investigate the prevalence of anaemia and its correlation with disease activity in IBD, in a large cohort of patients with matched full blood count (FBC) and FC data.

Methods Patients with confirmed IBD from the Edinburgh faecal calprotectin registry (EFCR) were identified. Where multiple FCs were available, the most recent result was taken as reference. Blood test results were obtained from the electronic record covering a period one month either side of the FC. The WHO criteria was used to define anaemia $(\mathrm{Hb}<130 \mathrm{~g} / \mathrm{L}$ in men, $<120 \mathrm{~g} / \mathrm{L}$ in women). A FC 